\title{
ERROS DE MEDICAÇÃO EM PEDIATRIA E ESTRATÉGIAS DE PREVENÇÃO: REVISÃO INTEGRATIVA
}

\author{
Bárbara Monteiro Volpatto ${ }^{1}$, Wiliam Wegner ${ }^{2}$, Luiza Maria Gerhardt ${ }^{3}$, Eva Neri Rubim Pedro ${ }^{3}$, Silvana da \\ Silva Cruz ${ }^{4}$, Larissa Edom Bandeira ${ }^{5}$
}

\begin{abstract}
RESUMO: Trata-se de revisão integrativa com o objetivo de analisar as estratégias, os incidentes de segurança e a etapa do processo medicamentoso para prevenção de eventos adversos na pediatria. Realizou-se a coleta entre novembro/2015 e fevereiro/2017, nas bases: Literatura Latino-Americana em Ciências de Saúde, Cumulative Index of Nursing and Allied Health Literature, U.S. National Library of Medicine e Web of Science. Utilizaram-se os descritores: erros de medicação, segurança do paciente e criança. Foram selecionados 27 artigos, publicados entre 2004 e 2016. A etapa do processo medicamentoso mais pesquisada foi a prescrição médica. As taxas de incidentes de segurança variaram entre $0,91 \%$ e $54 \%$, não sendo identificada padronização metodológica nos estudos. As estratégias de prevenção relatadas foram: uso da prescrição médica eletrônica, simulação clínica, protocolo de doses, e notificações de incidentes. É importante que a identificação de riscos e o planejamento das estratégias de prevenção subsidiem a análise global do processo medicamentoso.
\end{abstract}

DESCRITORES: Segurança do paciente; Erros de medicação; Criança; Enfermagem.

\section{MEDICATION ERRORS IN PEDIATRICS AND PREVENTION STRATEGIES: AN INTEGRATIVE REVIEW}

ABSTRACT: An integrative review was undertaken to analyze the strategies, safety incidents and the phase of the medication process intended to prevent adverse events in pediatrics. Data collection took place between November/2015 and February/2017 in the databases: Latin American Literature in Health Sciences, Cumulative Index of Nursing and Allied Health Literature, U.S. National Library of Medicine and Web of Science. The following descriptors were used: medication errors, patient safety and child. Twentyseven articles were selected, published between 2004 and 2016. The most investigated phase of the medication process was the drug prescription. The safety incident rates varied between $0.91 \%$ and $54 \%$. No methodological standardization could be identified in the studies. The reported prevention strategies were: use of electronic drug prescription, clinical simulation, dosing protocol and incident reports. It is important for the risk identification and planning of prevention strategies to support the global analysis of the medication process.

DESCRIPTORS: Patient safety; Medication errors; Child; Nursing.

\section{ERRORES DE MEDICACIÓN EN PEDIATRÍA Y ESTRATEGIAS DE PREVENCIÓN: REVISIÓN INTEGRATIVA}

RESUMEN: Esta es un revisión integrativa cuya finalidad fue analizar las estrategias, los incidentes de seguridad y la etapa del proceso medicamentoso para prevención de eventos adversos en la pediatría. Los datos fueron obtenidos entre noviembre/2015 y febrero/2017, en las bases: Literatura Latinoamericana en Ciencias de Salud, Cumulative Index of Nursingand Allied Health Literature, U.S. National Library of Medicine y Web of Science. Fueron utilizados los descriptores: errores de medicación, seguridad del paciente y niño. Se eligieron 27 artículos publicados entre 2004 y 2016. La etapa del proceso medicamentoso más investigada fue la prescripción médica. Las tajas de incidentes de seguridad variaron entre 0,91\% y 54\%, no siendo identificada estandarización metodológica en los estudios. Las estrategias de prevención relatadas fueron: uso de la prescripción médica electrónica, simulación clínica, protocolo de dosis, y notificaciones de incidentes. Es importante que la identificación de riesgos y el planeamiento de las estrategias de prevención subsidien el análisis global del proceso medicamentoso.

DESCRIPTORES: Seguridad del paciente; Errores de medicación; Niño; Enfermería.

${ }^{1}$ Enfermeira. Enfermeira no Hospital Mãe de Deus. Porto Alegre, RS, Brasil.

${ }^{2}$ Enfermeiro. Doutor em Enfermagem. Docente de Enfermagem da Universidade Federal do Rio Grande do Sul. Porto Alegre, RS, Brasil.

${ }^{3}$ Enfermeira. Doutora em Educação. Docente de Enfermagem da Universidade Federal do Rio Grande do Sul. Porto Alegre, RS, Brasil.

${ }^{4}$ Enfermeira. Doutoranda em Enfermagem. Universidade Federal do Rio Grande do Sul. Porto Alegre, RS, Brasil.

${ }^{5}$ Enfermeira. Especialista em Saúde da Criança. Enfermeira no Hospital de Clínicas de Porto Alegre. Porto Alegre, RS, Brasil.

Autor Correspondente:

Recebido: 29/02/2016

Wiliam Wegner

Universidade Federal do Rio Grande do Sul

Finalizado: 05/03/2017

Rua São Manoel, 963 - 90620-110 - Porto Alegre, RS, Brasil

E-mail:wiliam.wegner@ufrgs.br 


\section{INTRODUÇÃO}

O uso de medicamentos, atualmente, consiste no principal tratamento utilizado na terapêutica médica, e, consequentemente, os erros de medicação tornam-se mais frequentes ${ }^{(1)}$. Tais erros podem ocorrer em qualquer etapa do processo: prescrição, dispensação, armazenamento, preparo, entre outros. A prevenção desses envolve toda a equipe de saúde, destacando-se a de enfermagem, principal responsável pelas etapas de preparo e administração de medicamentos.

Considera-se erro de medicação qualquer evento evitável que, de fato ou potencialmente, possa interferir de forma inadequada no processo medicamentoso, podendo ou não provocar dano ao paciente ${ }^{(2)}$. Nesse contexto, entende-se Segurança do Paciente como a redução do risco de dano desnecessário associado ao cuidado de saúde. Esse dano pode ser físico, psicológico ou social; implicando prejuízo na estrutura ou função do corpo e/ou doenças, lesões, sofrimento, incapacidade ou morte. Assim, um incidente é um evento que pode ou não causar dano desnecessário ao paciente. Quando ocorre incidente com dano, tem-se um evento adverso ${ }^{(3)}$.

A preocupação com a segurança do paciente e com a qualidade do cuidado nos serviços de saúde mobilizou a Organização Mundial da Saúde (OMS), em 2004, a lançar a Aliança Mundial para a Segurança do Paciente. Esta objetivou melhorar a segurança na assistência, propondo medidas para reduzir riscos, organizando conceitos e definições sobre segurança do paciente e recomendando aos países maior atenção ao tema ${ }^{(4)}$.

Diante disso, pondera-se que as crianças são especialmente vulneráveis a danos, principalmente devido às peculiaridades do seu metabolismo como a idade, peso e estatura. Ademais, a deficiência de medicações padronizadas para pediatria torna o processo medicamentoso ainda mais suscetível a falhas ${ }^{(5)}$.

Promover ações e estratégias que visam aperfeiçoamento dos profissionais, padronização do processo e educação permanente são de suma importância para prevenir eventos adversos causados por erros de medicação(2). Quando o profissional entende o que é um evento adverso, suas causas e consequências, torna-se mais fácil admitir que o erro é possível e pode estar presente no cuidado. Esse entendimento é o primeiro passo para a prevenção ${ }^{(6)}$. Assim, saber o que se tem estudado e o que se está trabalhando nessa perspectiva mostra-se de extrema importância, justificando assim essa pesquisa.

Diante dessa problemática, essa revisão teve como questão de pesquisa: quais estratégias são propostas para prevenção de erros de medicação no cuidado à criança hospitalizada? Tendo como objetivo analisar as estratégias, os incidentes de segurança e a etapa do processo medicamentoso para prevenção de eventos adversos na pediatria.

\section{MÉTODO}

Trata-se de uma revisão integrativa (RI) da literatura que seguiu as seguintes etapas: formulação do problema, coleta de dados, avaliação dos dados, análise dos estudos e apresentação dos resultados ${ }^{(7)}$.

Na primeira etapa da RI, a seguinte questão norteadora foi proposta: quais estratégias são propostas para prevenção de erros de medicação no cuidado à criança hospitalizada?

Realizou-se a coleta entre novembro/2015 e fevereiro/2017, nas bases de dados: Literatura LatinoAmericana em Ciências de Saúde (LILACS), Cumulative Index of Nursing and Allied Health Literature (CINAHL), U.S. National Library of Medicine (PubMed) e Web of Science, selecionadas por serem importantes índices internacionais de literatura científica e técnica, com rigoroso padrão para indexação dos periódicos. Foram utilizados os Descritores em Ciências da Saúde (DeCS): erros de medicação, segurança do paciente e criança, nos idiomas inglês, espanhol e português de acordo com a base de dados, e utilizando o marcador booleano AND em cada cruzamento.

Os critérios de inclusão foram: ser artigo original - estudo primário; artigos científicos que tratassem da temática específica, erros de medicação, no título e resumo; textos escritos no idioma português, 
espanhol, inglês disponibilizados na íntegra e online e publicados no período entre 2004 e 2016.

Os critérios de exclusão foram: estudos de revisão, livros, teses e dissertações; estudos gerais sobre segurança do paciente; e estudos que não abordassem estratégias para prevenção de erros de medicação em pediatria.O fluxograma dos cruzamentos de descritores e resultados das buscas é apresentado na Figura 1 e seguiu as recomendações Prisma ${ }^{(8)}$.

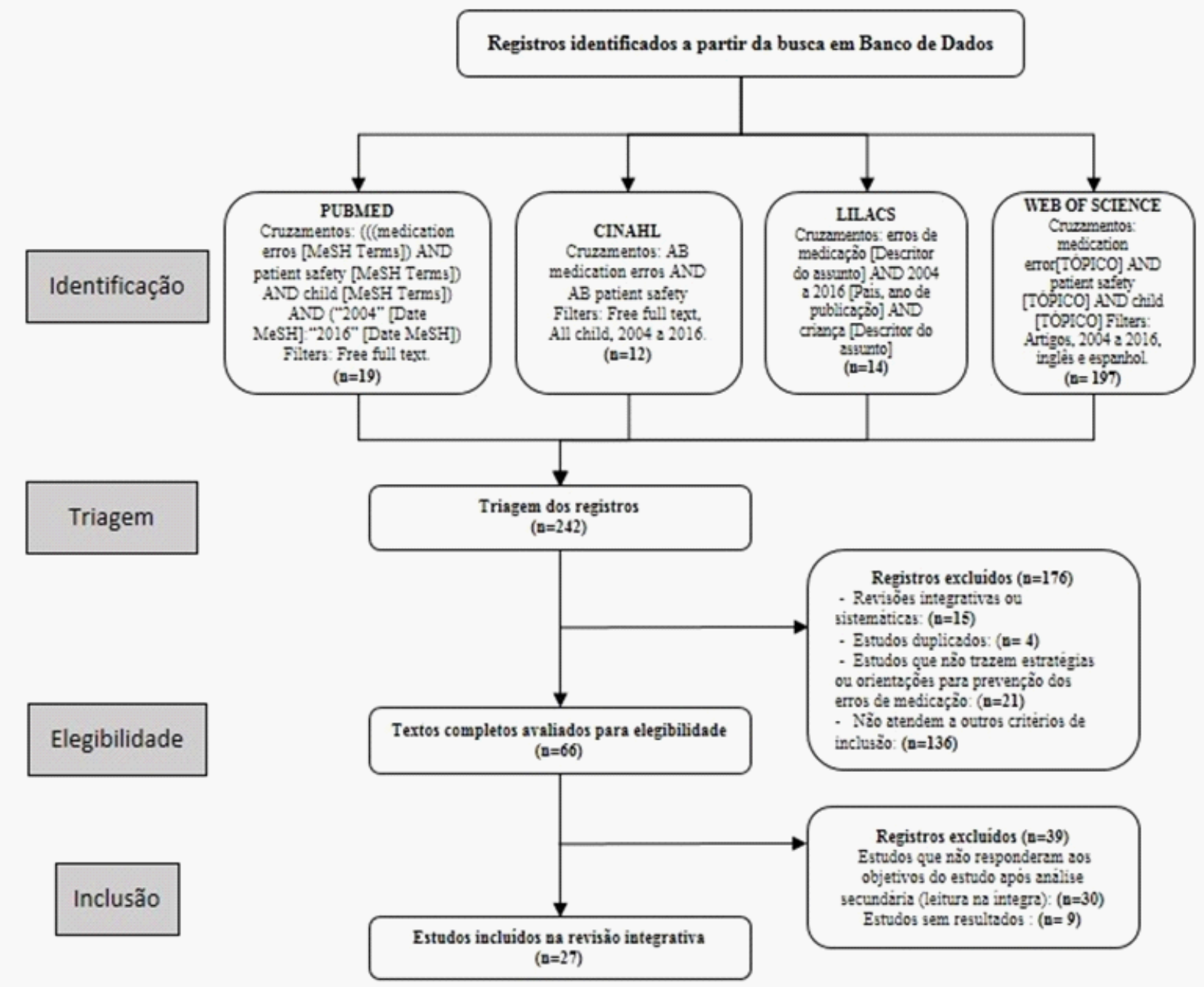

Figura 1 - Fluxograma da seleção de artigos científicos sobre prevenção de erros de medicação no cuidado à criança hospitalizada, conforme as bases de dados selecionadas. Porto Alegre, RS, Brasil, 2004-2016

Para análise e interpretação dos dados, realizou-se a síntese das informações extraídas dos artigos selecionados, buscando identificar neles quais estratégias são propostas para a prevenção dos erros de medicação, qual o número de incidentes e qual a etapa do processo de medicação em que ocorrem os erros. Utilizou-se instrumento composto dos seguintes itens: título do artigo; autores; periódico no qual o artigo foi publicado; país e ano; metodologia; estratégias para prevenção dos erros de medicação em pediatria; número de incidentes com medicação; e etapa do processo em que ocorreu o erro. Os dados extraídos e sintetizados são apresentados em dois quadros, um para a caracterização dos estudos e outro contemplando os resultados relacionados à questão norteadora.

\section{RESULTADOS}

Foram selecionados 27 artigos que discutem as estratégias propostas para prevenção de erros de medicação em pediatria, taxas de incidentes de segurança e etapas do processo medicamentoso em 
que ocorreram os erros. A caracterização dos estudos incluídos nesta RI é apresentada no Quadro 1.

Quadro 1 - Caracterização dos estudos segundo base de dados, ano, país, periódico, tipo de estudo e setor da pesquisa. Porto Alegre, RS, Brasil, 2004-2016. (continua)

\begin{tabular}{|c|c|c|c|c|c|c|}
\hline $\mathbf{N}^{\mathbf{o}}$ & $\begin{array}{l}\text { Base de } \\
\text { dados }\end{array}$ & Ano & País & Periódico & Tipo de estudo & Setor (local) da pesquisa \\
\hline 1 & LILACS & 2011 & Brasil & $\begin{array}{l}\text { Acta Paulista de São } \\
\text { Paulo }\end{array}$ & $\begin{array}{l}\text { Retrospectivo e } \\
\text { descritivo com } \\
\text { abordagem } \\
\text { quantitativa }^{(9)}\end{array}$ & Unidades pediátricas gerais \\
\hline 2 & LILACS & 2007 & Brasil & $\begin{array}{l}\text { Revista Brasileira de } \\
\text { Enfermagem }\end{array}$ & $\begin{array}{l}\text { Quase- } \\
\text { experimental(10) }\end{array}$ & $\begin{array}{l}\text { Infectologia, Unidade } \\
\text { Cirúrgica e de Terapia } \\
\text { Intensiva Pediátrica }\end{array}$ \\
\hline 3 & CINAHL & 2008 & EUA & $\begin{array}{l}\text { American Journal of } \\
\text { Health-System Pharmacy }\end{array}$ & $\begin{array}{l}\text { Quantitativo, } \\
\text { descritivo, } \\
\text { transversal }^{(11)}\end{array}$ & Unidades pediátricas gerais \\
\hline 4 & CINAHL & 2006 & EUA & $\begin{array}{l}\text { Journal of Pediatric } \\
\text { Nursing }\end{array}$ & $\begin{array}{l}\text { Quantitativo, } \\
\text { transversal }^{(12)}\end{array}$ & Unidades pediátricas gerais \\
\hline 5 & $\begin{array}{l}\text { WEB OF } \\
\text { SCIENCE }\end{array}$ & 2015 & EUA & $\begin{array}{l}\text { International Journal for } \\
\text { Quality in Health Care }\end{array}$ & $\begin{array}{l}\text { Prospectivo e de } \\
\text { intervenção }^{(13)}\end{array}$ & Unidades pediátricas gerais \\
\hline 6 & $\begin{array}{l}\text { WEB OF } \\
\text { SCIENCE }\end{array}$ & 2013 & EUA & Pediatric Blood Cancer & $\begin{array}{l}\text { Prospectivo e de } \\
\text { intervenção }^{(14)}\end{array}$ & Clínica Oncológica \\
\hline 7 & $\begin{array}{l}\text { WEB OF } \\
\text { SCIENCE }\end{array}$ & 2013 & EUA & $\begin{array}{l}\text { Emergency Medicine } \\
\text { Journal }\end{array}$ & Qualitativo(15) $^{(15)}$ & Emergência \\
\hline 8 & $\begin{array}{l}\text { WEB OF } \\
\text { SCIENCE }\end{array}$ & 2013 & Japão & $\begin{array}{l}\text { Journal of Food and Drug } \\
\text { Analysis }\end{array}$ & $\begin{array}{l}\text { Prospectivo e de } \\
\text { intervenção }^{(16)}\end{array}$ & Ambulatório de hospital \\
\hline 9 & $\begin{array}{l}\text { WEB OF } \\
\text { SCIENCE }\end{array}$ & 2013 & EUA & Pediatric Blood Cancer & $\begin{array}{l}\text { Transversal com } \\
\text { intervenção }^{(17)}\end{array}$ & Unidades pediátricas gerais \\
\hline 10 & $\begin{array}{l}\text { WEB OF } \\
\text { SCIENCE }\end{array}$ & 2013 & Canadá & $\begin{array}{l}\text { Journal of Pediatric } \\
\text { Nursing }\end{array}$ & $\begin{array}{l}\text { Descritivo, } \\
\text { prospectivo }^{(18)}\end{array}$ & Unidades pediátricas gerais \\
\hline 11 & $\begin{array}{l}\text { WEB OF } \\
\text { SCIENCE }\end{array}$ & 2012 & Espanha & $\begin{array}{l}\text { Archives of Disease in } \\
\text { Childhood }\end{array}$ & $\begin{array}{l}\text { Epidemiológico } \\
\text { transversal( }^{(19)}\end{array}$ & $\begin{array}{l}\text { Unidade pediátrica e } \\
\text { maternidade }\end{array}$ \\
\hline 12 & $\begin{array}{l}\text { WEB OF } \\
\text { SCIENCE }\end{array}$ & 2012 & Austrália & Pediatrics & $\begin{array}{l}\text { Prospectivo de } \\
\text { séries temporais }^{(20)}\end{array}$ & Unidades pediátricas gerais \\
\hline 13 & $\begin{array}{l}\text { WEB OF } \\
\text { SCIENCE }\end{array}$ & 2012 & Itália & $\begin{array}{l}\text { British Medical Journal } \\
\text { Open }\end{array}$ & $\begin{array}{l}\text { Quantitativo } \\
\text { comparativo com } \\
\text { intervenção }^{(21)} \\
\end{array}$ & Unidades pediátricas gerais \\
\hline 14 & $\begin{array}{l}\text { WEB OF } \\
\text { SCIENCE }\end{array}$ & 2011 & EUA & Pediatrics & $\begin{array}{l}\text { Prospectivo e de } \\
\text { intervenção }^{(22)}\end{array}$ & Unidades pediátricas gerais \\
\hline 15 & $\begin{array}{l}\text { WEB OF } \\
\text { SCIENCE }\end{array}$ & 2011 & EUA & $\begin{array}{l}\text { Applied Clinical } \\
\text { Informatics }\end{array}$ & $\begin{array}{l}\text { Estudo de análise } \\
\text { retrospectiva }^{(23)}\end{array}$ & Unidades de Internação \\
\hline 16 & $\begin{array}{l}\text { WEB OF } \\
\text { SCIENCE }\end{array}$ & 2011 & Irã & $\begin{array}{l}\text { Journal of Medical } \\
\text { Systems }\end{array}$ & $\begin{array}{l}\text { Prospectivo e de } \\
\text { intervenção }^{(24)}\end{array}$ & $\begin{array}{l}\text { Unidade de Terapia Intensiva } \\
\text { Neonatal }\end{array}$ \\
\hline 17 & $\begin{array}{l}\text { WEB OF } \\
\text { SCIENCE }\end{array}$ & 2009 & EUA & $\begin{array}{l}\text { Journal of Clinical } \\
\text { Nursing }\end{array}$ & $\begin{array}{l}\text { Qualitativo } \\
\text { descritivo }^{(25)}\end{array}$ & Unidades pediátricas gerais \\
\hline 18 & $\begin{array}{l}\text { WEB OF } \\
\text { SCIENCE }\end{array}$ & 2009 & Israel & Pediatrics & $\begin{array}{l}\text { Quantitativo } \\
\text { retrospectivo }^{(26)}\end{array}$ & $\begin{array}{l}\text { Unidade de Terapia Intensiva } \\
\text { Pediátrica }\end{array}$ \\
\hline 19 & $\begin{array}{l}\text { WEB OF } \\
\text { SCIENCE }\end{array}$ & 2008 & EUA & Pediatrics & Quantitativo $^{(27)}$ & Unidades pediátricas gerais \\
\hline 20 & $\begin{array}{l}\text { WEB OF } \\
\text { SCIENCE }\end{array}$ & 2008 & EUA & Pediatrics & $\begin{array}{l}\text { Prospectivo e de } \\
\text { intervenção }^{(28)} \\
\end{array}$ & Unidades pediátricas gerais \\
\hline 21 & $\begin{array}{l}\text { WEB OF } \\
\text { SCIENCE }\end{array}$ & 2006 & EUA & Pediatrics & $\begin{array}{l}\text { Quantitativo, } \\
\text { prospectivo }^{(29)}\end{array}$ & Unidades pediátricas gerais \\
\hline 22 & $\begin{array}{l}\text { WEB OF } \\
\text { SCIENCE }\end{array}$ & 2004 & EUA & $\begin{array}{l}\text { Quality and Safety in } \\
\text { Health Care }\end{array}$ & $\begin{array}{l}\text { Prospectivo e de } \\
\text { intervenção }^{(30)}\end{array}$ & $\begin{array}{l}\text { Unidade de Terapia Intensiva } \\
\text { Pediátrica }\end{array}$ \\
\hline
\end{tabular}


Cogitare Enferm. (22)1: e45132, 2017

\begin{tabular}{|c|c|c|c|c|c|c|}
\hline 23 & $\begin{array}{l}\text { WEB OF } \\
\text { SCIENCE }\end{array}$ & 2016 & EUA & $\begin{array}{l}\text { Journal of Pediatric } \\
\text { Surgery }\end{array}$ & $\begin{array}{l}\text { Metodológico } \\
\text { (criação de } \\
\text { instrumento) }\end{array}$ & Unidades Cirúrgicas \\
\hline 24 & $\begin{array}{l}\text { WEB OF } \\
\text { SCIENCE }\end{array}$ & 2016 & Canadá & $\begin{array}{l}\text { Journal of Pediatric } \\
\text { Nursing }\end{array}$ & $\begin{array}{l}\text { Quantitativo, } \\
\text { prospectivo }^{(32)}\end{array}$ & Unidades pediátricas gerais \\
\hline 25 & $\begin{array}{l}\text { WEB OF } \\
\text { SCIENCE }\end{array}$ & 2016 & EUA & Academic Pediatrics & $\begin{array}{l}\text { Quantitativo } \\
\text { (Delphi } \\
\text { modificado) }^{(33)}\end{array}$ & Ambulatório de hospital \\
\hline 26 & $\begin{array}{l}\text { WEB OF } \\
\text { SCIENCE }\end{array}$ & 2016 & Espanha & Enfermería Clínica & $\begin{array}{l}\text { Quantitativo, } \\
\text { descritivo, } \\
\text { transversal }\end{array}$ & Emergência Pediátrica \\
\hline 27 & $\begin{array}{l}\text { WEB OF } \\
\text { SCIENCE }\end{array}$ & 2016 & Canadá & $\begin{array}{l}\text { Journal of Evaluation in } \\
\text { Clinical Practice }\end{array}$ & $\begin{array}{l}\text { Quantitativo, } \\
\text { descritivo, } \\
\text { transversal(35) }\end{array}$ & Hospital Materno-Infantil \\
\hline
\end{tabular}

Dois estudos foram realizados no Brasil(9-10). A maioria das pesquisas sobre o tema proposto foi realizada no exterior, com destaque para Estados Unidos da América ${ }^{(11-15,17,22-23,25,27-31,33)}$. Com relação ao ano de publicação, há predomínio a partir do ano de $2011^{(9,13-24,31-35)}$. Unidades pediátricas gerais aparecem como maior foco dos estudos $(66,6 \%)$, entre elas, unidades de internação clínica e unidades cirúrgicas ${ }^{(9,11-13,17-23,25,27-29,31-32,35)}$. A Unidade de Terapia Intensiva (UTI) foi o setor isolado mais estudado $(18,5 \%)^{(2,10,24,26,30)}$.

Dos estudos que propuseram intervenções, observou-se que poucos seguiram uma proposta metodológica clássica com análise estatística. Estudos quantitativos, prospectivos e com intervenção ${ }^{(13-14,16,18,20-22,24,28-30,32)}$ foram $(48,1 \%)$. O periódico Pediatrics destacou-se por ter quatro publicações abordando a temática prevenção de erros de medicação em pediatria ${ }^{(20,22,26-29)}$. No Quadro 2 são apresentados os resultados que respondem ao objetivo desse estudo.

Quadro 2 - Caracterização dos estudos segundo, título do artigo, autores e resultados (estratégias de prevenção, número de incidentes e etapas do processo) nas publicações analisadas. Porto Alegre, RS, Brasil, 2004-2016 (continua)

\begin{tabular}{|c|c|c|}
\hline Título do Artigo & Autores & Resultados \\
\hline \multirow{3}{*}{$\begin{array}{l}\text { Notificação espontânea } \\
\text { de erros de medicação } \\
\text { em hospital universitário } \\
\text { pediátrico }^{(9)}\end{array}$} & \multirow{3}{*}{$\begin{array}{l}\text { Yamamoto MS, } \\
\text { Peterlini MAS, } \\
\text { Bohomol E. }\end{array}$} & $\begin{array}{l}\text { Estratégias de prevenção: Sistema de notificação e equipe } \\
\text { "Divisão de Enfermagem" responsável pelas notificações. }\end{array}$ \\
\hline & & $\begin{array}{l}\text { Número de incidentes: } 120 \text { ocorrências de erros de } \\
\text { medicação notificados, sendo } 45,8 \% \text { erros em } 2007 \text { e 54,2\% } \\
\text { em } 2008 \text {. }\end{array}$ \\
\hline & & $\begin{array}{l}\text { Etapas do processo: Omissão de dose em 2007; velocidade } \\
\text { da infusão em 2008. }\end{array}$ \\
\hline \multirow{3}{*}{$\begin{array}{l}\text { Redesenho de atividades da } \\
\text { enfermagem para redução } \\
\text { de erros de medicação em } \\
\text { pediatria }^{(10)}\end{array}$} & \multirow{3}{*}{$\begin{array}{l}\text { Yamanaka TI, } \\
\text { Pereira DG, } \\
\text { Pedreira MLG, } \\
\text { Peterlini MAS. }\end{array}$} & $\begin{array}{l}\text { Estratégias de prevenção: Construção de fluxograma de } \\
\text { prevenção e desenvolvimento de programa educacional. }\end{array}$ \\
\hline & & $\begin{array}{l}\text { Número de incidentes: Erros presentes em 21,1\% dos } 8152 \\
\text { medicamentos ou soluções analisadas. }\end{array}$ \\
\hline & & $\begin{array}{l}\text { Etapas do processo: Omissão de dose foi o tipo de erro de } \\
\text { maior ocorrência. }\end{array}$ \\
\hline \multirow[t]{3}{*}{$\begin{array}{l}\text { Characteristics of medication } \\
\text { errors and adverse drug events } \\
\text { in hospitals participating in } \\
\text { the California Pediatric Patient } \\
\text { Safety Initiative }{ }^{(11)}\end{array}$} & \multirow[t]{3}{*}{$\begin{array}{l}\text { Takata GS, } \\
\text { Taketomo CK, } \\
\text { Waite S. }\end{array}$} & $\begin{array}{l}\text { Estratégias de prevenção: Intervenção através de } 3 \\
\text { métodos: Pharmacy intervention medication errors (PIMEs) } \\
\text { a, Validated pediatric trigger methods (TADEs) }{ }^{\mathrm{b}} \text {, Voluntary } \\
\text { incident reports (VADEs) }{ }^{\mathrm{c}} \text {. }\end{array}$ \\
\hline & & $\begin{array}{l}\text { Número de incidentes: PIMEs } 349 \text { adverse drug events } \\
\text { (EAM)d identificados. TADEs } 79 \text { EAM identificados e, } \\
\text { VADEs } 278 \text { EAM identificados. }\end{array}$ \\
\hline & & Etapas do processo: não informado \\
\hline
\end{tabular}




\begin{tabular}{|c|c|c|}
\hline \multirow{3}{*}{$\begin{array}{l}\text { Harmful medication errors } \\
\text { in children: a } 5 \text {-year analysis } \\
\text { of data from the USP'S } \\
\text { MEDMARX Program }\end{array}$} & \multirow{3}{*}{$\begin{array}{l}\text { Hicks RW, } \\
\text { Becker SC, } \\
\text { Cousins DD. }\end{array}$} & $\begin{array}{l}\text { Estratégias de prevenção: Implantação de sistema que } \\
\text { avalia os registros de erros de medicação. }\end{array}$ \\
\hline & & $\begin{array}{l}\text { Número de incidentes: } 19.350 \text { registros de erros de } \\
\text { medicação durante } 5 \text { anos. }\end{array}$ \\
\hline & & $\begin{array}{l}\text { Etapas do processo: Erro de dose foi o tipo de erro com } \\
\text { maior ocorrência. }\end{array}$ \\
\hline \multirow[t]{3}{*}{$\begin{array}{l}\text { Electronic medication } \\
\text { reconciliation and medication } \\
\text { errors }^{(13)}\end{array}$} & \multirow{3}{*}{$\begin{array}{l}\text { Hron JD, Manzi } \\
\text { S, Dionne R, } \\
\text { Chiang VW, } \\
\text { Brostoff M, } \\
\text { Altavilla SA, et al. }\end{array}$} & $\begin{array}{l}\text { Estratégias de prevenção: Implementação de ferramenta } \\
\text { eletrônica de reconciliação de medicação e cumprimento } \\
\text { regular de relatórios para unidades de internação. }\end{array}$ \\
\hline & & $\begin{array}{l}\text { Número de incidentes: } 146 \text { erros de reconciliação de } \\
\text { medicação em admissões. }\end{array}$ \\
\hline & & Etapas do processo: não informado \\
\hline \multirow[t]{3}{*}{$\begin{array}{l}\text { The use of a checklist in a } \\
\text { pediatric oncology } \text { clinic }^{(14)}\end{array}$} & \multirow{3}{*}{$\begin{array}{l}\text { McLean TW, } \\
\text { White GM, } \\
\text { Bagliani AF, } \\
\text { Lovato JF. }\end{array}$} & $\begin{array}{l}\text { Estratégias de prevenção: Implantação de checklist em } \\
\text { unidade ambulatorial oncológica. }\end{array}$ \\
\hline & & $\begin{array}{l}\text { Número de incidentes: Erros de prescrição medicamentosa } \\
\text { do prontuário eletrônico reduziram de } 21 \% \text { no } 1^{\circ} \text { mês para } \\
12 \% \text { no } 5^{\circ} \text { mês. }\end{array}$ \\
\hline & & Etapas do processo: não informado \\
\hline \multirow{3}{*}{$\begin{array}{l}\text { Reported medication events in } \\
\text { a pediatric emergency research } \\
\text { network: sharing to improve } \\
\text { patient safety }^{(15)}\end{array}$} & \multirow{3}{*}{$\begin{array}{l}\text { Shaw KN, Lillis } \\
\text { KA, Ruddy RM, } \\
\text { Mahajan PV, } \\
\text { Lichenstein R, } \\
\text { Olsen CS, et al. }\end{array}$} & $\begin{array}{l}\text { Estratégias de prevenção: Sistema de relatórios de } \\
\text { incidentes para qualificar e quantificar as notificações de } \\
\text { eventos adversos. }\end{array}$ \\
\hline & & $\begin{array}{l}\text { Número de incidentes: } 597 \text { (19\%) erros de medicação de } \\
3.106 \text { relatórios de incidentes analisados em um ano. }\end{array}$ \\
\hline & & Etapas do processo: Maior ocorrência de erros de dose. \\
\hline \multirow{3}{*}{$\begin{array}{l}\text { The effect of a computerized } \\
\text { pediatric dosing } \\
\text { decision support system on } \\
\text { pediatric dosing error }^{(16)}\end{array}$} & \multirow{3}{*}{$\begin{array}{l}\text { Jing-Yi Hou, } \\
\text { Kuei-Ju Cheng, } \\
\text { Kuan-Jen Bai, } \\
\text { Hsiang-Yin } \\
\text { Chen, Wen-Hao } \\
\text { Wud, You-Meei } \\
\text { Lin, et al. }\end{array}$} & $\begin{array}{l}\text { Estratégias de prevenção: Implantação de sistema } \\
\text { computadorizado de prescrição médica e apoio à decisão } \\
\text { de dosagem pediátrica. }\end{array}$ \\
\hline & & $\begin{array}{l}\text { Número de incidentes: Taxa de erros de prescrição } \\
\text { medicamentosa reduziu de } 2,23 \% \text { para } 0,66 \% \text { após a } \\
\text { intervenção. }\end{array}$ \\
\hline & & Etapas do processo: não informado \\
\hline \multirow{3}{*}{$\begin{array}{l}\text { Chemotherapy medication } \\
\text { errors in a pediatric cancer } \\
\text { treatment center: prospective } \\
\text { characterization of error } \\
\text { types and frequency and } \\
\text { development of a quality } \\
\text { improvement initiative to lower }_{\text {the error rate }}^{(17)}\end{array}$} & \multirow[t]{3}{*}{$\begin{array}{l}\text { Watts RG, } \\
\text { Parsons K. }\end{array}$} & $\begin{array}{l}\text { Estratégias de prevenção: Implantação de sistema } \\
\text { informatizado de prescrição, checagem e pedido de } \\
\text { quimioterápicos. }\end{array}$ \\
\hline & & Número de incidentes: Taxa de erro global reduziu 50\%. \\
\hline & & $\begin{array}{l}\text { Etapas do processo: Tipos de erros mais comuns: dosagem } \\
\text { (overdose e/ou subdose), unidades de medida, droga, } \\
\text { técnica de administração ou via incorretos. }\end{array}$ \\
\hline \multirow{3}{*}{$\begin{array}{l}\text { The relationship between the } \\
\text { nursing work environment } \\
\text { and the occurrence of } \\
\text { reported pediatric medication } \\
\text { administration errors: a pan } \\
\text { Canadian study }\end{array}$} & \multirow{3}{*}{$\begin{array}{l}\text { Sears K, } \\
\text { O`Brien-Pallas L, } \\
\text { Stevens B, } \\
\text { Murphy GT. }\end{array}$} & $\begin{array}{l}\text { Estratégias de prevenção: Elaboração de questionário para } \\
\text { coleta de dados de erros de medicação. }\end{array}$ \\
\hline & & $\begin{array}{l}\text { Número de incidentes: } 372 \text { erros foram relatados no } \\
\text { período de } 3 \text { meses. }\end{array}$ \\
\hline & & Etapas do processo: não informado \\
\hline \multirow[t]{3}{*}{$\begin{array}{l}\text { Impact of clinical pharmacist } \\
\text { interventions in reducing } \\
\text { pediatric prescribing errors }\end{array}$} & \multirow{3}{*}{$\begin{array}{l}\text { Fernández- } \\
\text { Llamazares CM, } \\
\text { Calleja- } \\
\text { Hernandez MA, } \\
\text { Manrique- } \\
\text { Rodriguez S, } \\
\text { Pérez-Sanz C, } \\
\text { Duran-García } \\
\text { E,Sanjurjo-Saez } \\
\text { M. }\end{array}$} & $\begin{array}{l}\text { Estratégias de prevenção: Farmacêuticos clínicos analisaram } \\
\text { as atividades de farmacêuticos pediátricos referentes às } \\
\text { prescrições em pediatria. }\end{array}$ \\
\hline & & $\begin{array}{l}\text { Número de incidentes: } 1.391 \text { intervenções - de } 1.357 \text { erros } \\
\text { de prescrição, } 833 \text { foram erros de dosagem. }\end{array}$ \\
\hline & & Etapas do processo: Erros de prescrição. \\
\hline
\end{tabular}


Long-term reduction in adverse drug events: an evidencebased improvement mode/ ${ }^{(20)}$
Gazarian M, Graudins LV.

Lago P, Bizzarri

G, Scalzotto

F, Parpaiola

A, Amigoni A,

Putoto G, et al.
Estratégias de prevenção: Implantação de diretrizes para prescrição pediátrica multiprofissional segura.

Número de incidentes: Total de erros de medicação diminuiu, em 4 anos, de 4,51 por 100 prescrições, para 2,78 por 100 prescrições.

Etapas do processo: Prescrição médica.

Estratégias de prevenção: Implantação de ferramenta proativa para avaliar riscos, identificar possíveis falhas e priorizar medidas de prevenção.

Número de incidentes: não informado

Etapas do processo: Prescrição e preparação das drogas surgiram como etapas mais vulneráveis a erros.

Estratégias de prevenção: Implantação de pacote de intervenções para melhorar a cultura da segurança.

Número de incidentes: Taxa de eventos adversos medicamentosos caiu $42 \%$ no trimestre final do projeto (22,4 EAM por 1000 pacientes-dia).

Etapas do processo: não informado

Estratégias de prevenção: Alertas de dosagem em sistema de prescrição médica eletrônica com suporte para decisões clínicas para pacientes pediátricos hospitalizados.

Número de incidentes: De 1.024 alertas de dosagem disparados pelo sistema, 91\% foram para sobredosagem e 9\% para subdosagem.

Etapas do processo: Prescrição médica.

Estratégias de prevenção: Sistema informatizado de prescrição e suporte de decisão clínica.

Número de incidentes: 53\% (antes da implantação da prescrição médica eletrônica com suporte de decisão clínica) e 34\% (após a implantação).

Etapas do processo: Prescrição médica (cálculo de dose).

Estratégias de prevenção: Compreensão da prática da enfermagem, dos facilitadores e barreiras em relação ao processo de dupla checagem independente de medicações em serviços pediátricos.

Número de incidentes: não informado

Etapas do processo: Preparo e administração de medicamentos.

Kadmon G, Bron-Harlev

Computerized order entry with limited decision support to prevent prescription errors in a PICU (26)

E, Nahum E, Schiller O, Haski G, Shonfeld T.

Estratégias de prevenção: Sistema de prescrição eletrônica e suporte de decisão clínica.

Número de incidentes: 5.000 prescrições analisadas, 273 (5,5\%) continham erros de prescrição. Após implementação, taxa de erros reduziu em $83 \%$.

Etapas do processo: Prescrição médica.

Estratégias de prevenção: Implementação de prescrição médica eletrônica.

Número de incidentes: Incidência de eventos adversos evitáveis de medicação foi de 7,9 antes da implementação para 6,5 após implementação.

Etapas do processo: Prescrição médica. 


\begin{tabular}{|c|c|c|}
\hline \multirow{3}{*}{$\begin{array}{l}\text { Reevaluating the safety profile } \\
\text { of pediatrics: a comparison } \\
\text { of computerized adverse } \\
\text { drug event surveillance and } \\
\text { voluntary reporting in the } \\
\text { pediatric environment }{ }^{28)}\end{array}$} & \multirow{3}{*}{$\begin{array}{lr}\text { Ferranti } & \mathrm{J}, \\
\text { Horvath } & \mathrm{MM}, \\
\text { Cozart } & \mathrm{H}, \\
\text { Whitehurst } & \mathrm{J}, \\
\text { Eckstrand J. } & \end{array}$} & $\begin{array}{l}\text { Estratégias de prevenção: Uso concomitante de } \\
\text { sistema informatizado de vigilância de eventos adversos } \\
\text { medicamentosos e de sistema de notificação voluntária de } \\
\text { incidentes de segurança. }\end{array}$ \\
\hline & & $\begin{array}{l}\text { Número de incidentes: } 1,8 \text { incidentes por } 1.000 \text { pacientes } \\
\text { pediátricos-dia segundo o sistema de notificação voluntária } \\
\text { de incidentes de segurança; } 1,6 \text { incidentes por } 1.000 \\
\text { pacientes-dia segundo o sistema informatizado de vigilância } \\
\text { de eventos adversos medicamentosos. }\end{array}$ \\
\hline & & Etapas do processo: Prescrição e administração. \\
\hline \multirow{3}{*}{$\begin{array}{l}\text { Risk reduction for adverse } \\
\text { drug events through sequential } \\
\text { implementation of patient } \\
\text { safety initiatives in a children's } \\
\text { hospital }{ }^{29)}\end{array}$} & \multirow{3}{*}{$\begin{array}{l}\text { Leonard MS, } \\
\text { Cimino M, Shaha } \\
\text { S, McDougal S, } \\
\text { Pilliod J, Brodsky } \\
\text { L. }\end{array}$} & $\begin{array}{l}\text { Estratégias de prevenção: Implementação sequencial de } \\
\text { iniciativas para educação e mudança de comportamento do } \\
\text { profissional prescritor. }\end{array}$ \\
\hline & & $\begin{array}{l}\text { Número de incidentes: A redução do risco absoluto de } \\
\text { erros de prescrição foi de } 38 \text { por } 100 \text { prescrições, com uma } \\
\text { redução do risco relativo de } 49 \% \text {. }\end{array}$ \\
\hline & & Etapas do processo: Prescrição médica. \\
\hline \multirow[t]{3}{*}{$\begin{array}{l}\text { Design of a safer approach to } \\
\text { intravenous drug infusions: } \\
\text { failure mode effects analysis }{ }^{(30)}\end{array}$} & \multirow{3}{*}{$\begin{array}{l}\text { Apkon M, } \\
\text { Leionard J, } \\
\text { Probst L, DeLizio } \\
\text { L, Vitale R. }\end{array}$} & $\begin{array}{l}\text { Estratégias de prevenção: Desenvolvimento de um } \\
\text { conjunto de processos padronizados para infusão contínua } \\
\text { de medicamentos. }\end{array}$ \\
\hline & & Número de incidentes: não informado \\
\hline & & Etapas do processo: Prescrição, preparo e administração. \\
\hline \multirow[t]{3}{*}{$\begin{array}{l}\text { Implementation of a pediatric } \\
\text { surgical quality improvement } \\
\text { (QI)-driven M\&M conference }{ }^{(31)}\end{array}$} & \multirow[t]{3}{*}{$\begin{array}{l}\text { Cromeens B, } \\
\text { Brilli R, Kurtovic } \\
\text { K, Kenney B, } \\
\text { Nwomeh } \\
\text { Besner GE. }\end{array}$} & $\begin{array}{l}\text { Estratégias de prevenção: para todos os tipos de falhas, } \\
\text { foco na educação; nos casos de falhas de profissionais, } \\
\text { otimização da comunicação, estabelecimento de critérios } \\
\text { para consultoria interdisciplinar, resolução de problemas } \\
\text { com equipamentos, remoção de medicamentos de alto risco } \\
\text { de protocolos de procedimentos, modificação de conjuntos } \\
\text { de ordens/prescrições, reestruturação de transferências de } \\
\text { cuidados pelos médicos. }\end{array}$ \\
\hline & & $\begin{array}{l}\text { Número de incidentes: } 107 \text { pacientes. Foram identificadas } \\
142 \text { falhas: } 78,9 \% \text { erros individuais e } 21,1 \% \text { fallhas no sistema. } \\
\text { Relato de caso de uma criança que teve insuficiência } \\
\text { respiratória devido ao uso de capacaína spray (anestésico } \\
\text { orofaríngeo) para passagem de sonda nasogástrica no setor } \\
\text { de radiologia. A análise mostrou que foi uma falha no sistema } \\
\text { - falta de protocolo para uso do medicamento. }\end{array}$ \\
\hline & & Etapas do processo: prescrição médica. \\
\hline \multirow[t]{3}{*}{$\begin{array}{l}\text { The relationship between } \\
\text { nursing experience and } \\
\text { education and theoccurrence of } \\
\text { reported pediatric medication } \\
\text { administration } \text { errors }^{(32)}\end{array}$} & \multirow[t]{3}{*}{$\begin{array}{l}\text { Sears K, O'Brien- } \\
\text { Pallas L, Stevens } \\
\text { B, Murphy GT. }\end{array}$} & $\begin{array}{l}\text { Estratégias de prevenção: inserir a notificação de incidentes } \\
\text { de segurança na formação de enfermeiros (p. ex., simulação } \\
\text { de evento adverso no laboratório); utilizar a notificação de } \\
\text { erros como sistema de feedback para melhorar o processo } \\
\text { de administração de medicamentos (instituição pró ativa); } \\
\text { construção de cultura de confidencialidade da notificação; } \\
\text { educar enfermeiros com menos experiência em determinada } \\
\text { unidade. }\end{array}$ \\
\hline & & $\begin{array}{l}\text { Número de incidentes: média de } 29,18(\mathrm{DP}=9,86) \text { erros em } \\
\text { cada unidade durante o período de } 3 \text { meses de coleta dos } \\
\text { dados com base no auto relato de enfermeiros (limitação } \\
\text { identificada pelos autores: subnotificação de erros pelos } \\
\text { enfermeiros por motivos diversos). Mínimo: um erro; } \\
\text { máximo: } 43 \text { erros. }\end{array}$ \\
\hline & & Etapas do processo: não informado \\
\hline
\end{tabular}




\begin{tabular}{|c|c|c|}
\hline \multirow{3}{*}{$\begin{array}{l}\text { Evaluating the potential severity } \\
\text { of look-alike, sound-alike drug } \\
\text { substitution errors in children }{ }^{(33)}\end{array}$} & \multirow{3}{*}{$\begin{array}{l}\text { Basco WT, } \\
\text { Garner,D SS, } \\
\text { Ebeling M, } \\
\text { Freeland KD, } \\
\text { Hulsey TC, } \\
\text { Simpson K. }\end{array}$} & Estratégias de prevenção: alertas eletrônicos automáticos. \\
\hline & & $\begin{array}{l}\text { Número de incidentes: para } 207 \text { pares }(34 \%) \text { de } \\
\text { medicamentos com grafia ou som semelhantes, estimou-se } \\
\text { que nenhum paciente recebeu o medicamento trocado. } \\
\text { Para } 298 \text { pares }(49 \%) \text { de medicamentos com grafia ou } \\
\text { som semelhantes, estimou-se que o total cumulativo } \\
\text { de sujeitos que receberam o medicamento trocado foi } \\
3610 \text { (aproximadamente } 1 \text { erro/dia ao longo de } 10 \text { anos). } \\
\text { Em contraste, entre os restantes } 103 \text { pares (17\%) de } \\
\text { medicamentos com grafia ou som semelhantes, estimou- } \\
\text { se que } 97163 \text { sujeitos receberam medicamento trocado (27 } \\
\text { erros potenciais/dia ao longo de } 10 \text { anos). }\end{array}$ \\
\hline & & Etapa do processo: prescrição e dispensação. \\
\hline \multirow[t]{3}{*}{$\begin{array}{l}\text { Conocimiento de las enfermeras } \\
\text { de las dosis de medicamentos } \\
\text { en urgencias de pediatria }{ }^{(34)}\end{array}$} & \multirow{3}{*}{$\begin{array}{l}\text { Guerrero- } \\
\text { Márqueza } \\
\text { G, Martínez- } \\
\text { Serranob } \\
\text { A, Míguez- } \\
\text { Navarroa C, } \\
\text { López-Mirónc } \\
\text { JA, Espartosa- } \\
\text { Larrayadd M. }\end{array}$} & $\begin{array}{l}\text { Estratégias de prevenção: educação, elaboração e } \\
\text { implementação de protocolos para manejo e dose de } \\
\text { medicamentos. }\end{array}$ \\
\hline & & Número de incidentes: não informado \\
\hline & & $\begin{array}{l}\text { Etapa do processo: administração de medicamentos (dose } \\
\text { certa). }\end{array}$ \\
\hline \multirow[t]{3}{*}{$\begin{array}{l}\text { Medication errors room: } \\
\text { a simulation to assess the } \\
\text { medical, nursing and pharmacy } \\
\text { staffs' ability to identify errors } \\
\text { related to the medication-use } \\
\text { system }^{(35)}\end{array}$} & \multirow[t]{3}{*}{$\begin{array}{l}\text { Daupin J, } \\
\text { Atkinson S, } \\
\text { Bédard P, Pelchat } \\
\text { V, Lebel D, } \\
\text { Bussières JF. }\end{array}$} & $\begin{array}{l}\text { Estratégias de prevenção: conscientização dos profissionais } \\
\text { sobre riscos do processo medicamentoso por meio de } \\
\text { simulação/simulação como instrumento para educação } \\
\text { dos profissionais. Disponibilização permanente de jogo de } \\
\text { simulação online. }\end{array}$ \\
\hline & & Número de incidentes: não informado \\
\hline & & Etapa do processo: prescrição, dispensação e administração. \\
\hline
\end{tabular}

Legenda: a Pharmacy intervention medication errors (PIMEs): intervenção da farmácia nos erros de medicação; ${ }^{\mathrm{b}}$ Validated pediatric trigger method (TADEs): método validado de detecção de potenciais eventos adversos pediátricos em prontuários; ${ }^{c} V o l u n t a r y$ incident reports (VADEs): notificações voluntárias de incidentes; ${ }^{\mathrm{d}}$ Adverse drug events (EAM): eventos adversos medicamentosos.

\section{- DISCUSSÃO}

\section{Estratégias para prevenção de erros de medicação}

A identificação das estratégias de prevenção foi o objetivo principal desse estudo. A implantação ou melhoria no sistema eletrônico de prescrição médica foi o tipo de estratégia mais investigada $(25,9 \%)$ nos estudos avaliados, mostrando redução nas taxas de erros de medicação após as intervenções ${ }^{(16-17,23-24,26-27,33)}$. O uso dessa ferramenta reduz erros de legibilidade e facilita a comunicação entre as equipes. Em estudo realizado com acadêmicos de enfermagem para identificar o conhecimento deles sobre segurança do paciente, a prescrição eletrônica foi uma das estratégias mais referidas como uma das maneiras de prevenção e redução de erros de medicação(36).

Os estudos usaram como aliados à prescrição eletrônica, um sistema de apoio à decisão clínica de dosagem, alertas eletrônicos automáticos para nomes semelhantes e a implementação do protocolos para doses pediátricas. É um sistema informatizado, semelhante a uma calculadora, onde se inserem peso e idade da criança e o sistema calcula a dose da medicação, alertando quando há overdose ou subdose, por exemplo (16,23-24,26,33-34). Em outro estudo, foi implantada uma padronização de cálculos de dose, tempo e programação da bomba de infusão para medicamentos de infusão contínua em uma Unidade de Terapia Intensiva Pediátrica (UTIP), como estratégia de prevenção de eventos adversos ${ }^{(30)}$.

A padronização e protocolização para manejo de doses pediátricas foi foco de estudo realizado em Madri/Espanha e mostrou que um terço dos profissionais de enfermagem não confere a dose usual 
prescrita $^{(34)}$.

Em uma pesquisa realizada em hospital universitário nos EUA, foi desenvolvida uma ferramenta eletrônica para aperfeiçoar a reconciliação de medicamentos na admissão do paciente. A ferramenta mostra, lado a lado, a lista de medicamentos em uso pelo paciente antes da admissão e a lista de medicamentos prescritos no momento da internação. Essa estratégia possibilitou identificar e quantificar a redução dos incidentes após a intervenção, através da análise de relatórios de erros de medicação ${ }^{(13)}$.

A dupla checagem é uma ação essencial para prevenção de erros durante as etapas mais críticas do processo medicamentoso. No entanto, destacou-se em apenas um estudo, que avaliou o entendimento dos enfermeiros acerca desse procedimento ${ }^{(25)}$. Além disso, alguns estudos buscaram implementar estratégias de prevenção como uso de checklist, protocolo clínico, fluxograma na administração de medicamentos e protocolos para manejo de doses ${ }^{(10,14,34)}$. Tais ações propunham reestruturar e organizar o processo medicamentoso com o objetivo de reduzir os erros de medicação.

O sistema de notificação espontânea de eventos adversos de medicação revela-se como uma estratégia de prevenção nos estudos analisados ${ }^{(9,11-12,15,28)}$. Pesquisa realizada nos EUA, durante cinco anos, concluiu que analisar relatórios de erros de medicação é uma forma de encorajar que outros possam aprender com os erros, evitando a repetição desses no futuro ${ }^{(12)}$. Outros estudos buscaram ações para implementar estratégias de rastreamento de erros através de ferramentas (trigger tool) ou de questionários específicos ${ }^{(11,18)}$. Identificaram-se recomendações importantes, como: aumento do nível de preparação profissional para enfermeiros pediátricos; melhora da qualidade da comunicação acerca da administração segura de medicamentos entre a equipe interdisciplinar e da equipe com as famílias; e reconhecimento dos desafios para administração segura de medicamentos ${ }^{(18)}$. A reestruturação de processos a partir das falhas, com ações educativas e melhoria da comunicação entre a equipe, a inclusão da notificação na formação do enfermeiro, com o uso da simulação em laboratórios, o desenvolvimento da cultura da confidencialidade e do feedback, a importância da conscientização sobre os erros em jogos virtuais online, favorecem, também, o desenvolvimento de estratégias educativas para prevenção de erros de medicação no cuidado à saúde da criança ${ }^{(31-32,35)}$.

Quanto à participação dos profissionais de saúde, destacam-se os farmacêuticos como profissionais fundamentais no processo medicamentoso e na prevenção dos eventos adversos. Um estudo desenvolvido na Espanha demonstrou o impacto significativo das intervenções do farmacêutico clínico na prevenção da ocorrência de erros de prescrição ${ }^{(19)}$.

A educação continuada sobre administração de medicamentos foi implementada em um estudo recente no Brasil. Verificou-se redução da omissão de doses e aumento da taxa de identificação de erros na UTIP após a intervenção ${ }^{(10)}$. Ademais, estratégias educacionais, mediadas por grupos focais ou websites educacionais e jogos de simulação online, demonstraram ser intervenções importantes para melhoria da comunicação multidisciplinar, para desenvolvimento da cultura de segurança e para redução das taxas de incidentes envolvendo medicações ${ }^{(20,25,29,34)}$.

A análise dos estudos mostrou uma deficiência de estratégias de prevenção que visam reduzir os erros na etapa da administração de medicamentos. Reforça-se a importância de instituírem-se barreiras em todas as etapas do processo, mas a administração, como última barreira, necessita de maiores investigações, de modo que se compreendam as principais fragilidades nesse processo e se busquem estratégias de prevenção.

\section{Número de incidentes de segurança do paciente com medicação}

Os estudos analisados não apresentam uma padronização metodológica, o que dificulta comparações e replicações. O estabelecimento de um protocolo de pesquisa padronizado na área de incidentes de segurança do paciente pediátrico proporcionaria maior consistência nos dados e conclusões.

As taxas de incidentes com erros de medicação variaram, nesse estudo de RI, entre 0,91\% e 54\%, relatadas em pesquisas que tiveram de três meses a 10 anos de duração ${ }^{(9,16,33)}$. Apenas um estudo, realizado nos EUA, não demonstrou redução significativa nas taxas de erros de medicação após 
implementação de sistema de prescrição eletrônica ${ }^{(27)}$.

Observou-se que, dos 27 estudos analisados, 10 apresentaram taxas de incidentes com medicação pré e pós intervenção. A maioria apresentou redução dos incidentes após a intervenção proposta $^{(10,13-14,16-17,20,22,24,26,29)}$. Estudo recente, realizado no Japão, mostrou redução na taxa de incidentes de erros de medicação de $2,23 \%$ para $0,66 \%$ pós intervenção em um período de 14 meses. A taxa de correção de quase-falhas aumentou 10 vezes após a implementação do sistema de prescrição médica eletrônica com suporte para decisões clínicas ${ }^{(16)}$. O uso da simulação clínica e jogos virtuais online para detectar erros foi mensurado em pesquisa realizada no Canadá, demonstrando que $78,4 \%$ dos farmacêuticos obtiveram taxa de resposta correta para erros de medicação, enfermeiros e médicos atingiram índice semelhante $(67 \%)$. Concluiu-se que a conscientização sobre os riscos é uma ação educativa fundamental para fortalecer o uso seguro de medicamentos ${ }^{(35)}$.

Pesquisa realizada nos Estados Unidos da América, que avaliou 608 pares de medicamentos com nomes semelhantes, trouxe dados preocupantes quanto à taxa de incidentes, estimando que em somente 34\% dos pares não houve administração de medicamento trocado. Em 49\% (298 pares) dos fármacos com grafia ou som semelhante, o medicamento foi trocado em 3610 ocasiões, apresentando uma taxa cumulativa de 01/dia ao longo de 10 anos. Em 17\%, a taxa estimada foi de 27 erros/dia, representando 97163 pacientes que receberam fármacos trocados ${ }^{(33)}$.

Em 40,7\% dos estudos, somente as taxas de incidentes foram mensuradas após a implantação das ações educativas ${ }^{(9,11-12,15,18-19,23,28,31-32,35)}$. Um sistema de notificações espontâneas de um hospital universitário pediátrico de São Paulo demonstrou 120 ocorrências de erros de medicação, sendo 45,8\% em 2007 e 54,2\% em $2008^{(9)}$. O aumento das notificações é considerado um bom resultado em termos de desenvolvimento da cultura de segurança.

O conhecimento e acompanhamento das taxas de incidentes com erros de medicação devem subsidiar a implantação de estratégias de prevenção, demonstrando sua eficácia e oportunizando ajustes. As estatísticas envolvendo o processo medicamentoso contribuem para dimensionar o problema e dar visibilidade à importância de desenvolver estratégias educativas para prevenção dos erros de medicação.

\section{Etapas do processo medicamentoso com maior registro de erros}

A prescrição é a etapa do processo medicamentoso que apresentou mais eventos adversos (40,7\%) (11,19-21,23-24,26-27,31,33,35). Os erros de medicação em pacientes pediátricos têm relação direta com a necessidade de dosagem baseada no peso exato. Um sistema de prescrição médica eletrônica, combinado a um sistema de apoio à decisão clínica e alertas eletrônicos automáticos, são ferramentas importantes para a prevenção de erros ${ }^{(23,33)}$.

Erros que envolvem dose errada de medicação também foram observados em quatro estudos ${ }^{(12,15,17,34)}$. Um estudo com analgésicos opioides e antidiabéticos mostrou elevado número de erros de dosagem ${ }^{(12)}$.

Em dois estudos realizados no Brasil, a omissão de dose teve destaque. As causas podem estar relacionadas à falta de atenção, sobrecarga de trabalho, cansaço, estresse e falta de funcionários, entretanto, devem ser consideradas as falhas nos sistemas de prevenção ${ }^{(9-10)}$.

Um estudo apresentou as três etapas do processo medicamentoso (prescrição, dispensação e administração), enfatizando que o problema dos erros estava relacionado ao sistema. Os participantes da simulação detectaram corretamente $67,5 \%$ dos erros relacionados ao sistema de medicação ${ }^{(35)}$.

Em 37\% das pesquisas analisadas, não foi o objetivo identificar a etapa do processo medicamentoso em que os erros acontecem ${ }^{(13-14,16,18,22,25,28-30,32)}$. Os erros de prescrição foram o objeto de estudo na maioria das pesquisas analisadas. Reforça-se que todas as etapas do processo medicamentoso estão inter-relacionadas e todos os profissionais de saúde, em especial a equipe de enfermagem, têm responsabilidades durante esse processo. 
No presente estudo, identificou-se como principais estratégias de prevenção de erros de medicação à criança hospitalizada, a implantação de sistema de prescrição médica eletrônica, uso da simulação clínica, a implantação do protocolos para manejo de doses e sistemas de notificações de eventos adversos.

A taxa de incidentes envolvendo o processo de medicação mostrou-se elevada, e a etapa da prescrição médica foi objeto de estudo na maioria das pesquisas. Tais resultados apontam para a necessidade de se desenvolverem estudos sobre estratégias para prevenção de eventos adversos em todas as etapas do processo medicamentoso voltado à criança hospitalizada.

A análise dos estudos selecionados fortalece a importância de envolvimento de toda a equipe multiprofissional na conscientização e desenvolvimento da cultura de notificação e de reconhecimento dos riscos/fatores contribuintes para falhas no processo/sistema de medicamentos.

Considera-se que este estudo teve algumas limitações referentes às pesquisas bibliográficas, uma delas quanto à escolha do descritor "criança", o qual se mostrou muito abrangente, apresentando um número alto de estudos. Assim como a grande diversidade metodológica dos artigos selecionados, o que dificultou comparações aprofundadas entre os estudos.

\section{REFERÊNCIAS}

1. Belela ASC, Pedreira MLG, Peterlini MAS. Erros de medicação em pediatria. Rev. bras.enferm. 2011;64(3):563-9.

2. Ministério da Saúde (BR). Agência Nacional de Vigilância Sanitária. Protocolo de segurança na prescrição, uso e administração de medicamentos. Brasília: Anvisa; 2013.

3. Organização Mundial de Saúde. Estrutura conceitual da classificação internacional sobre segurança do doente. Relatório técnico final. Lisboa: Organização Mundial de Saúde; 2011. Co-publicado pela Direção-Geral da Saúde.

4. Agência Nacional de Vigilância Sanitária (BR). Boletim Informativo - Segurança do Paciente e Qualidade nos Serviços de Saúde. 2011;1(1):1-12.

5. Harada MJCS, Chanes DC, Kusahara DM, Pedreira MLG. Segurança na administração de medicamentos em pediatria. Acta paul.enferm. 2012;25(4):639-42.

6. Wegner W, Pedro ENR. Patient safety in care circumstances: prevention of adverse events in the hospitalization of children. Rev. Latino-Am. Enfermagem. 2012;20(3):427-34.

7. Cooper HM. The integrative research review: a systematic approach. Thousand Oaks, CA: Sage Publications; 1984.

8. Urrútia G, Bonfill X. Declaración PRISMA: una propuesta para mejorar la publicación de revisiones sistemáticas y metaanálisis. Med Clin (Barc). 2010;135(11):507-11.

9. Yamamoto MS, Peterlini MAS, Bohomol E. Notificação espontânea de erros de medicação em hospital universitário pediátrico. Acta paul.enferm. 2011;24(6):766-71.

10. Yamanaka TI, Pereira DG, Pedreira MLG, Peterlini MAS. Redesenho das atividades de enfermagem para redução de erros de medicação em pediatria. Rev. bras. enferm. 2007;60(2):190-6.

11. Takata GS, Taketomo CK, Waite S,California Pediatric Patient Safety Initiative. Characteristics of medication errors and adverse drug events in hospitals participating in the California Pediatric Patient Safety Initiative. Am J Health Syst Pharm. 2008;65(1):2036-44.

12. Hicks RW, Becker SC, Cousins DD. Harmful medication errors in children: a 5-year analysis of data from the USP's MEDMARXR Program. J Pediatr Nurs. 2006;21(4):290-8. 
13. Hron JD, Manzi S, Dionne R, Chiang VW, Brostoff M, Altavilla SA, et al. Electronic medication reconciliation and medication errors. Int J Qual Health Care. 2015;27(4):314-9.

14. McLean TW, White GM, Bagliani AF, Lovato JF. The use of a checklist in a pediatric oncology clinic. Pediatr Blood Cancer. 2013;60(11):1855-9.

15. Shaw KN, Lillis KA, Ruddy RM, Mahajan PV, Lichenstein R, Olsen CS, et al Reported medication events in a pediatric emergency research network: sharing to improve patient safety. Emerg Med J. 2013;30(10):815-9.

16. Hou JY, Cheng KJ, Bai KJ, Chen HY, Wu WH, Lin YM, et al The effect of a computerized pediatric dosing decision support system on pediatric dosing errors. J Food Drug Anal. 2013;21(3):286-91.

17. Watts RG, Parsons K. Chemotherapy medication errors in a pediatric cancer treatment center: prospective characterization of error types and frequency and development of a quality improvement initiative to lower the error rate. Pediatr Blood Cancer. 2013;60(8):1320-4.

18. Sears K, O'Brien-Pallas L, Stevens B, Murphy GT. The relationship between the nursing work environment and the occurrence of reported paediatric medication administration errors: a pan Canadian study. J Pediatr Nurs. 2013;28(4):351-6.

19. Fernández-Llamazares CM, Calleja-Hernandez MA, Manrique-Rodriguez S, Pérez-Sanz C, Duran-García E, Sanjurjo-Saez M. Impact of clinical pharmacist interventions in reducing paediatric prescribing errors. Arch Dis Child. 2012;97(6):564-8.

20. Gazarian M, Graudins LV. Long-term reduction in adverse drug events: an evidence-based improvement model. Pediatrics. 2012;129(5):e1334-42.

21. Lago P, Bizzarri G, Scalzotto F, Parpaiola A, Amigoni A, Putoto G, et al.Use of FMEA analysis to reduce risk of errors in prescribing and administering drugs in paediatric wards: a quality improvement report. BMJ Open. 2012;2(6):e001249.

22. Tham E, Calmes HM, Poppy A, Eliades AB, Schlafly SM, Namtu KC, et al.Sustaining and spreading the reduction of adverse drug events in a multicenter collaborative. Pediatrics. 2011;128(2):e438-45.

23. Perlman SL, Fabrizio L, Shaha SH, Magid SK. Response to medication dosing alerts for pediatric inpatients using a computerized provider order entry system. Appl Clin Inform. 2011;2(4):522-33.

24. Kazemi A, Ellenius J, Pourasghar F, Tofighi S, Salehi A, Amanati A, et al. The effect of computerized physician order entry and decision support system on medication errors in the neonatal ward: experiences from an Iranian teaching hospital. J Med Syst. 2011;35(1):25-37.

25. Dickinson A, McCall E, Twomey B, James N. Paediatric nurses' understanding of the process and procedure of double-checking medications. J Clin Nurs. 2010;19(5-6):728-35.

26. Kadmon G, Bron-Harlev E, Nahum E, Schiller O, Haski G, Shonfeld T. Computerized order entry with limited decision support to prevent prescription errors in a PICU. Pediatrics. 2009;124(3):935-40.

27. Walsh KE, Landrigan CP, Adams WG, Vinci RJ, Chessare JB, Cooper MR, et al. Effect of computer order entry on prevention of serious medication errors in hospitalized children. Pediatrics. 2008;121(3):421-7.

28. Ferranti J, Horvath MM, Cozart H, Whitehurst J, Eckstrand J. Reevaluating the safety profile of pediatrics: a comparison of computerized adverse drug event surveillance and voluntary reporting in the pediatric environment. Pediatrics. 2008;121(5):e1201-7.

29. Leonard MS, Cimino M, Shaha S, McDougal S, Pilliod J, Brodsky L. Risk reduction for adverse drug events through sequential implementation of patient safety initiatives in a children's hospital. Pediatrics. 2006;118(4):e1124-9.

30. Apkon M, Leonard J, Probst L, DeLizio L, Vitale R. Design of a safer approach to intravenous drug infusions: failure mode effects analysis. Qual Saf Health Care. 2004;13(4):265-71.

31. Croomens B, Brilli R, Kurtovic K, Kenney B, Nwomeh B, Besner GE. Implementation of pediatric surgical quality improvement (QI)- driven M\&M conference. JPediatrSurg. 2016;51(1):137-42. 
32. Sears K, O’brien-Pallas L, Stevens B, Murphy GT. The relationship between nursing experience and education and the occurrence of reported pediatric medication administration errors. JPediatr Nurs. 2016;31(4):e283-90.

33. Basco Jr WT, Gamer SS, Ebeling M, Freeland KD, Hulsey TC, Simpson K. Evaluating the potential severity of look-alike, sound-alike drug substitution errors in children. Acad Pediatr. 2016;16(2):183-91.

34. Guerrero-Márquez G, Martínez-Serrano A, Míguez-Navarro C, López-Mirón JA, Espartosa-Larrayad M. Conocimiento de las enfermeras de las dosis de medicamentos en urgencias de pediatría. Enferm Clin. 2016;26(4):213-9.

35. Daupin J, Atkinson S, Bédard P, Pelchat V, Lebel D, Bussières JP. Medication errors room: a simulation to assess the medical, nursing and pharmacy staffs ability to identify errors related to the medication-use system. JEvalClin Pract.2016;22(6):907-16.

36. Bogarin DF, Zanetti ACB, Brito MFP, Machado JP, Gabriel CS, Bernardes A. Segurança do Paciente: conhecimento de alunos de graduação em enfermagem. Cogitare Enferm. 2014;19(3):491-7. 